\title{
Airway Considerations in Pediatric Neurosurgical Patients
}

\author{
Hemant Bhagat ${ }^{1,3}$ Summit Dev Bloria ${ }^{1,3}$ Sonia Kapil ${ }^{2} \quad$ Narender Kaloria $^{3}$ \\ ${ }^{1}$ Neuroanaesthesia Division, Post Graduate Institute of Medical \\ Education \& Research, Chandigarh, India \\ ${ }^{2}$ Neuroanaesthesia Division, Paras Hospital Panchkula, Haryana, India

\begin{abstract}
Address for correspondence Summit Dev Bloria, MD, DM, Post Graduate Institute of Medical Education \& Research, Chandigarh, India (e-mail: summitbloria13@gmail.com).
\end{abstract}

${ }^{3}$ Anaesthesia Department, Post Graduate Institute of Medical

Education \& Research, Chandigarh, India

Indian J Neurosurg:2020;9:179-182
Abstract
Keywords
- pediatric patients' airway
- airway
- neurosurgery

Managing airway in pediatric neurosurgical patients can prove to be challenging. In this article, we wish to touch upon the specific airway considerations to be kept in mind while caring for these patients during neurosurgeries.

\section{Introduction}

Care of pediatric patients during neurosurgery is one of the most challenging tasks for the anesthesiologists. The reasons for this are many: decreased physiological reserve in these patients, airway issues, long duration of surgery, possibility of extreme blood loss, positioning during the surgery, etc. Among all these factors, one factor which is often not given its due importance but nevertheless can lead to disastrous consequences is management of airway in pediatric patients undergoing neurosurgery. A pediatric airway differs from adult airway in the following ways:

1. Children have large heads, so neck extension during mask ventilation and intubation becomes difficult.

2. Nasal cavities are narrow, predisposing to nasal obstruction.

3. Tongue is comparatively large, falls back, and obstructs the airway.

4. Mandible is thin and underdeveloped.

5. Epiglottis is large and omega-shaped.

6. Larynx is funnel-shaped and situated higher up in the airway, which makes a straight laryngoscope blade more practical for intubation.

7. Subglottis is the narrowest part of the airway.

8. Infants suffer from deoxygenation rapidly due to highoxygen consumption.

9. Dynamic airway collapse of the upper airways occurs during forceful inspiration.

DOI https://doi.org/

$10.1055 / \mathrm{s}-0040-1713341$

ISSN 2277-954X.
10. Ventilation is primarily diaphragmatic, and "bucket handle" action is not possible due to horizontal ribs. Also, bulky abdominal organs or a gas-filled stomach impede effective ventilation.

In this article, we wish to touch upon the specific considerations to be kept in mind while caring for these patients during neurosurgeries.

\section{Selection of Endotracheal Tube-Type and Size}

A) Cuffed versus uncuffed endotracheal tube (ETT)-Use of uncuffed tubes lowers the resistance to breathing, allows easy suctioning, and prevents trauma to subglottic region. For procedures of long duration, use of pediatric cuffed tubes is advisable; nowadays, microcuff ETT are also available for the same. Their use allows employment of lower fresh gas flows, reduces air pollution, avoids multiple intubation attempts, and provides better end tidal $\mathrm{CO}_{2}$ monitoring.

B) Appropriate size and length of ETT-For determination of appropriate size and length of the endotracheal tube, various formulas have been suggested. Some of the most commonly used among them are:

- The internal diameter (ID) of an ETT: (Age in years/4) + 4 .

- The ID of ETT = Diameter of the fifth finger. 
The following formulas have been used to suggest the depth of ETT insertion from teeth:

Depth of tube: Height (in $\mathrm{cm}) / 10+5$.

or

Weight (in kgs) $/ 5+12$.

or

Three times the ID from the alveolar ridge.

or

(Age in years/2) +12 .

or

Advance the ETT into a bronchus and then withdraw it by $2 \mathrm{~cm}$.

Specific airway concerns can occur in pediatric neurosurgical patients because of one or more of the following reasons:

\section{Airway issues due to primary pathology and site of pathology.}

2. Airway issues due to positioning of the patient intraoperatively.

3. Airway issues due to lack of accessibility of airway during the surgery.

We discuss all these issues one by one.

\section{Airway Issues Due to Primary Pathology and Site of Pathology}

The children can prove to be difficult to ventilate or intubate directly as a result of their primary pathology affecting the airway. This primary pathology can affect:

- The cranium/intracranial compartment(e.g., hydrocephalus, encephalocele, craniosynostosis, etc.)

- The craniovertebral junction (e.g., Chiari malformations, atlanto-axial dislocation, etc.)

- Spine disorders (e.g., neural tube defects).

Hydrocephalus-Defined as an abnormal increase in cerebrospinal volume, hydrocephalus can occur due to an increase in cerebrospinal fluid (CSF) production or a disturbance in flow or absorption of CSF. Increased CSF volume in these children leads to abnormal enlargement of head circumference. This enlarged cranium can be difficult to mask ventilate and intubate. ${ }^{1}$ Hence, a difficult airway cart must be available while managing airway of these patients. The enlarged head interferes with ideal head position for mask ventilation and laryngoscopy. To counteract this, a towel roll should be placed under the shoulders of the child. These children can undergo VP shunt procedure, endoscopic third ventriculostomy (ETV), or choroid plexectomy for management of hydrocephalus. In VP shunt, the neck of the child is rotated to one side to facilitate burr-hole creation at Frazier's point. This neck rotation can lead to displacement of ETT, and one must make it a point to auscultate both lungs after final position for surgery has been made. Also, excessive neck rotation can impede venous return from the head. In ETV, the head is positioned at a higher level compared with the heart, predisposing to occurrence of venous air embolism.

Craniosynostosis-Craniosynostosis is associated with genetic syndromes like Apert and Crouzon. Crouzon syndrome is an autosomal dominant genetic disorder characterized by hypertelorism, proptosis, maxillary hypoplasia, prognathism, and hydrocephalus. Apert syndrome children manifest choanal atresia, a deviated nasal septum, a narrowed nasopharynx, a thick long soft palate, lateral palatal swellings, tongue-based airway obstruction, and a tracheal cartilage sleeve. These children have difficult airways. In patients who have undergone surgery for craniosynostosis previously, intubation may be more difficult, as a result of the altered relationships between the maxilla and mandible and reduced temporomandibular joint movement. ${ }^{2}$ Many syndromic craniosynostosis patients suffer from obstructive sleep apnea. Many institutes use special ETTs like south-facing Ring-Adair-Elwyn tubes while caring for these patients.

Chiari malformation-Chiari malformations are a group of defects associated with congenital caudal displacement of the cerebellum and brainstem. They are subdivided into types 1 to 4.

Type 1 -It is themost common type, characterized by downward displacement of cerebellar tonsils into the upper cervical canal through the foramen magnum. Vocal cord paralysis with a compromised airway and inspiratory stridor during infancy is a well-recognized complication. ${ }^{3}$

Type 2-It is also known as the Arnold Chiari malformation. Usually associated with a lumbosacral spinal myelomeningocele, it is characterized by displacement of the medulla, fourth ventricle and cerebellar vermis through the foramen magnum. Although its etiology of CM-II is not well understood, it has been suggested that both open neural tube defect and incomplete spinal occlusion lead to CSF leakage from the fetal spinal canal and ventricular system. The resulting lack of ventricular CSF distention precludes the full development of the normal size posterior fossa which, in turn, leads to the caudal displacement of the rapidly developing cerebellum into the spinal canal along with the brainstem.

It is associated with brainstem dysfunction, leading to inspiratory stridor, central apnea and cyanosis. ${ }^{4}$

Type 3-An occipital and/or high cervical encephalocele associated with type 2 Chiari malformation.

Type 4-Severe hypoplasia of cerebellum without displacement of the cerebellum through the foramen magnum.

Patients with Chiari malformation and brainstem surgery may exhibit intermittent postoperative apnea, vocal cord paralysis, or other irregularities before resuming a stable respiratory pattern. ${ }^{5}$ Vocal cord palsies is said to be caused by brainstem herniation with traction on vagal fibers innervating vocal cord adductor muscles.

Neural tube defects-These include spina bifida occulta, meningocele, encephalocele, and meningomyelocele. While intubating these patients, a donut should be kept around the swelling, so that it does not get ruptured. Alternatively, these patients can be intubated in the lateral position. In patients with giant encephaloceles, one may have to take the child to the edge of the table with his or her head hanging for intubation. ${ }^{6}$

\section{Craniovertebral Junction Abnormalities}

Craniovertebral junction abnormalities can be congenitallike malformations of occiput/atlas/axis or developmental/ 
acquired abnormalities like Paget's disease, achondroplasia, Down's syndrome, rheumatoid arthritis, traumatic, etc. These patients may have instability of cervical spine and there is a possible risk of neurological deficit developing during the process of securing the airway.

In these patients, the preoperative neurodeficit must be documented, and their parents explained the possibility of intubation, leading to further neurodeficit. Awake fiberoptic intubation in children may not be possible in all cases, and as a general rule, a videolaryngoscope should be used if available. Laryngoscopy should be performed in neutral position and manual in-line stabilization should be used.

\section{Airway Issues Due to Positioning of the Patient Intraoperatively}

The commonly employed positions in pediatric patients are as follows:

a. Supine position-Most commonly used for CSF diversion procedures and supratentorial surgeries. As described earlier, excessive neck rotation should be avoided, and final ETT confirmed before draping. Also, the ETT should be fixed in the nondependent corner of the mouth, so that oral secretions do not soil the adhesive tapes used for fastening. ${ }^{7}$

b. Prone position-Used in posterior fossa surgeries, spine surgeries and neural tube defect surgeries. For posterior fossa surgeries, the child's neck is flexed, and this flexion can result in endobronchial intubation due to distal migration of the ETT. Intraoral kinking of ETT has also been reported due to excessive neck flexion. ${ }^{8}$ Hence, using reinforced ETTs may be considered for children undergoing these surgeries. Many people prefer nasotracheal tubes while managing children in prone position. However, nasotracheal intubations are contraindicated in patients with choanal atresia, basilar skull fractures, transsphenoidal surgeries, and concurrent sinusitis.

Abdomen must be free to allow adequate ventilation. Significant airway edema and postoperative obstruction can occur after prolonged prone procedures or those involving significant blood loss with high-volume replacement. These patients should be kept in head-up position postoperatively for resolution of airway edema.

C. Sitting position-Used for posterior fossa surgeries, upper cervical spine and pineal tumors. Airway advantages of sitting position include increase in functional residual capacity (FRC), better access to airway, and unimpeded ventilation. However, excessive neck flexion in sitting position can cause macroglossia and myelopathy.

d. Lateral position-Use of lateral position is uncommon in pediatric neurosurgical patients. In this position, the compliance of downside lung decreases.

\section{Airway Issues Due to Lack of Accessibility of Airway during the Surgery}

The airway remains inaccessible to the anesthesiologist during neurosurgery. The face of the child gets covered with surgical drapes, and it is not possible to visualize the ventilator circuit and the ETT. Hence, it becomes necessary to properly fix the endotracheal tube to the patient using either adhesive tape, sutures or wiring. Also, one must be ready with a rescue plan in cases of accidental extubation.

Unplanned intraoperative extubations are said to have an event rate of $<0.3 \%$; with lower patient age, patient manipulation, and anesthesia depth as risk factors. ${ }^{9}$

\section{Adjuncts for Pediatric Airway Management}

1. Nasopharyngeal airway-It is better tolerated than an oral airway in patients with intact airway reflexes. However, adenoids and tonsils can get traumatized during insertion and cause bleeding. Luo et al suggested that "nasopharyngeal airway could better maintain unobstructed upper airway in neurosurgery patients during the anesthesia recovery period with advantages such as small circulatory effect, good tolerance and fewer complications," although the study did not include pediatric patients. ${ }^{10}$

2. Oropharyngeal airway-It prevents airway obstruction caused by the large tongue falling backward. It should however only be used when the child's gag reflex is depressed to prevent occurrence of coughing and laryngospasm. The correct size is estimated by holding the airway next to the patient's mouth. The tip of airway should lie above the angle of the mandible

\section{Role of Supraglottic Airways in Pediatric Neuroanesthesia}

Supraglottic airways (SGA) like I-gel and laryngeal mask airway (LMA) have revolutionized airway management, playing a specific role in pediatric neuroanesthesia also. SGA can be used to ventilate children in situations when it is not able to intubate them. Also, SGAs can prove to be an alternative to intubation in some short neurosurgical procedures. ${ }^{11,12}$ Choosing the right size of SGA is important, and it depends upon the weight of the child as follows:

\begin{tabular}{|l|l|}
\hline I-gel size & Body weight $(\mathbf{k g})$ \\
\hline 1 & $2-5$ \\
\hline 1.5 & $5-12$ \\
\hline 2 & $10-25$ \\
\hline 2.5 & $25-35$ \\
\hline
\end{tabular}

\section{Child with Recent Upper Respiratory Tract Infection}

A child with recent upper respiratory tract infection (URTI) was said to be at a higher risk of laryngospasm, atelectasis, pneumonia, bronchospasm, postintubation croup, and desaturation episodes. The safest bet in this situation for a child who needs to undergo an elective procedure consists of postponing the procedure. However, it should be remembered that bronchial hyperresponsiveness may take as long as 6 weeks to return to normal; recently, some authors have 
suggested that if these children are optimized, most of them can have uneventful surgeries. ${ }^{13}$ To conclude, the decision to proceed with anesthesia and surgery should be the result of a multidisciplinary discussion involving anesthetist, surgeon, and family, keeping in mind the welfare of the child as the first priority.

\section{Weaning and Extubation in Pediatric Neurosurgical Patients}

Inside the operation theater, one must aim for smooth emergence and uneventful extubation of patients. Premature extubation of the trachea can lead to laryngospasm or inadequate respiratory efforts, which can lead to hypoxia and cardiac arrest. Inside ICU, one must be more guarded before extubating children.

Role of corticosteroids-Up to $37 \%$ of critically ill pediatric patients may develop glottic or subglottic edema due to intubation, resulting in stridor upon extubation. ${ }^{14}$ It has been suggested that prophylactic corticosteroids in children reduces the incidence of postextubation stridor and may also decrease the incidence of reintubation. ${ }^{15}$ An often used prophylactic corticosteroid regime consists of dexamethasone $0.5 \mathrm{mg} / \mathrm{kg}$ (up to a maximum of $10 \mathrm{mg}$ ), with the first dose 6 to 12 hour before extubation, and then every 6 hours for six doses in total. ${ }^{16,17}$

Leak test-This test is used to rule out airway edema and is recommended before extubating children who have undergone prolonged surgeries in prone position. While ventilating the child with an airway pressure of 15 to $20 \mathrm{~mm} \mathrm{Hg}$, there should be an appreciable air leak caused due to space between the ETT and the tracheal wall. The absence of an awir leak indicates a high-risk of upper airway obstruction, and extubation should be postponed in these cases.

\section{Laryngospasm}

Sustained closure of vocal cords causes inability to ventilate the patient. It can be triggered by manipulation of airway (e.g., laryngoscopy or suctioning), presence of blood or secretions in airway. Asthmatic children and those with recent URTI are more predisposed to develop laryngospasm. Treatment consists of giving continuous positive airway pressure with $100 \%$ oxygen, administering propofol to deepen plane of anesthesia $(0.5 \mathrm{mg} / \mathrm{kg})$, and calling for help. Succinylcholine $(0.1 \mathrm{mg} / \mathrm{kg})$ is the drug of choice if propofol fails to relieve laryngospasm.

\section{Conflict of Interest}

None declared.

\section{References}

1 Vagyannavar R, Bharti V, Hashim M. Difficult airway in a case of gross hydrocephalus for shunt surgery. Anesth Essays Res 2017;11(4):1109-1111

2 de Beer D, Bingham R. The child with facial abnormalities. Curr Opin Anaesthesiol 2011;24(3):282-288

3 Allsopp GM, Karkanevatos A, Bickerton RC. Abductor vocal fold palsy as a manifestation of type one Arnold Chiari malformation. J Laryngol Otol 2000;114(3):221-223

4 Choi SS, Tran LP, Zalzal GH. Airway abnormalities in patients with Arnold-Chiari malformation. Otolaryngol Head Neck Surg 1999;121(6):720-724

5 Saigal D, Ganjoo P, Sharma MU, Singh D. Managing tracheal extubation in infants with stridor and congenital neuraxial anomalies. J Pediatr Neurosci 2016;11(4):335-337

6 Murthy PS, Kalinayakanahalli Ramkrishnappa SK. Giant occipital encephalocele in an infant: A surgical challenge. J Pediatr Neurosci 2019;14(4):218-221

7 Krane EJ, Phillip BM, Yeh KK, Domino KB, Anaesthesia for paediatric neurosurgery. In: Smith RM, Mototyama EK, Davis PJ, eds. Smith's Anaesthesia for Infants and Children. 7th ed. Philadelphia: Mosby; 2006 651-84

8 Bharti N, Bala I. Kinking of endotracheal tube during posterior fossa surgery. Indian J Anaesth 2010;54(2):172-173

9 Wagner KM, Raskin JS, Carling NP, et al. Unplanned intraoperative extubations in pediatric neurosurgery: analysis of case series to increase patient safety. World Neurosurg 2018;115:e1-e6

10 Luo LL, Zhou LX, Chen LJ, Lü S, Huang W, Luo D. [The application of nasopharyngeal airway in nurosurgery patients during anesthesia recovery]. Sichuan Da Xue Bao Yi Xue Ban 2013;44(4):689-692

11 Agarwal A, Shobhana N. LMA in neurosurgery. Can J Anaesth 1995;42(8):750

12 Fan $\mathrm{CH}$, Peng $\mathrm{B}$, Zhang FC. Influence of laryngeal mask airway (LMA) insertion anesthesia on cognitive function after microsurgery in pediatric neurosurgery. Eur Rev Med Pharmacol Sci 2017;21(4, Suppl):37-42

13 Rachel Homer J, Elwood T, Peterson D, Rampersad S. Risk factors for adverse events in children with colds emerging from anesthesia: a logistic regression. Paediatr Anaesth 2007;17(2):154-161

14 Kemper KJ, Benson MS, Bishop MJ. Predictors of postextubation stridor in pediatric trauma patients. Crit Care Med 1991;19(3):352-355

15 Khemani RG, Randolph A, Markovitz B. Corticosteroids for the prevention and treatment of post-extubation stridor in neonates, children and adults. Cochrane Database Syst Rev 2009; (3):CD001000

16 Tellez DW, Galvis AG, Storgion SA, Amer HN, Hoseyni M, Deakers TW. Dexamethasone in the prevention of postextubation stridor in children. J Pediatr 1991;118(2):289-294

17 Anene O, Meert KL, Uy H, Simpson P, Sarnaik AP. Dexamethasone for the prevention of postextubation airway obstruction: a prospective, randomized, double-blind, placebo-controlled trial.CritCare Med 1996;24(10):1666-1669 